\title{
Measuring Insulin Resistance in Humans
}

\author{
Vandhna R. Sharma ${ }^{\mathrm{a}}$ Samantha T. Matta ${ }^{\mathrm{a}}$ Morey W. Haymond ${ }^{\mathrm{b}}$ \\ Stephanie T. Chung a \\ ${ }^{a}$ National Institute of Diabetes and Digestive and Kidney Diseases, National Institutes of Health, Bethesda, MD, USA; \\ ${ }^{\text {b}}$ Baylor College of Medicine, Houston, TX, USA
}

\section{Keywords}

Insulin resistance $\cdot$ Insulin assay $\cdot$ History $\cdot$ Insulin sensitivity · Insulin action

\begin{abstract}
Background: Insulin resistance is a pathophysiological condition associated with diabetes and cardiometabolic diseases that is characterized by a diminished tissue response to insulin action. Our understanding of this complex phenomenon and its role in the pathogenesis of cardiometabolic diseases is rooted in the discovery of insulin, its isolation and purification, and the challenges encountered with its therapeutic use. Summary: In this historical perspective, we explore the evolution of the term "insulin resistance" and demonstrate how advances in insulin and glucose analytics contributed to the recognition and validation of this metabolic entity. We identify primary discoveries which were pivotal in expanding our knowledge of insulin resistance, the challenges in measurement and interpretation, contemporary techniques, and areas of future exploration. Key Message: Measurements of insulin resistance are important tools for defining and treating cardiometabolic diseases. Accurate quantification of this pathophysiological entity requires careful consideration of the assumptions and pitfalls of the methodological techniques and the historical and clinical context when interpreting and applying the results.
\end{abstract}

(C) 2021 The Author(s)

Published by S. Karger AG, Basel

karger@karger.com www.karger.com/hrp

Karger $\stackrel{\text { ' }}{=}$
(C) 2021 The Author(s)

Published by S. Karger AG, Basel

This is an Open Access article licensed under the Creative Common Attribution-NonCommercial-4.0 International License (CC BY-NC) (http://www.karger.com/Services/OpenAccessLicense), applicable to the online version of the article only. Usage and distribution for commercial purposes requires written permission.

\section{Introduction}

The last century has witnessed monumental discoveries in insulin and the diagnosis and management of diabetes. Each advancement progressively increased our understanding of the mechanisms behind insulin resistance and its central role in the pathogenesis of cardiometabolic diseases. As we "stand on the shoulders of giants," we must acknowledge the origins of our current use of the term "insulin resistance," to deepen our appreciation of the many contributing factors for this metabolic condition and spur us to explore innovative treatments to reduce the disease burden. Primary discoveries that were important in the conceptualization of insulin resistance included measurement of plasma glucose; the discovery, isolation, purification, and crystallization of insulin; radioimmunoassay (RIA) measurements of serum insulin; the standardization of insulin dosing; the development of recombinant human insulin; and a number of molecular modifications to the insulin structure. Detailed cataloging of these early discoveries is beyond the scope of this article but has been recently highlighted in several centennial reviews [1-3]. In this historical perspective, we focus on the evolution of measuring insulin resistance from the early twentieth century to our contemporary understanding of this metabolic phenomenon and its association with excess adiposity.

Correspondence to:

Stephanie T. Chung, stephanie.chung@ nih.gov 


\section{First Reports of Insulin Resistance}

Marked intra- and interindividual variabilities in glycemic response to insulin were first observed soon after the discovery, isolation, and clinical use of insulin for the treatment of diabetes in $1922[4,5]$. At the time, insulin was in short supply and in high demand because of the labor-intensive process of isolating and purifying insulin from whole pancreata. As physicians worked to obtain insulin for their sickest patients, publications of specific individual characteristics associated with poor insulin response became more widespread, despite the growing concerns of impurities in the extracts $[6,7]$. These reports marked the first documentation of diminished response to insulin and were defined as the inability to lower glucosuria even after administrating "large" doses of these crude preparations of insulin. Initially, "large" was relative, but the definition was grounded in Macleod's experiments, in which 32 units/day of insulin was sufficient to maintain euglycemia in a 16-pound female depancreatized dog [4]. Based on these trials, the amount of insulin required to maintain euglycemia in a depancreatized 150 lb man was extrapolated, and a "refractory" insulin response was defined as the administration of $\geq 200$ units/ day of insulin in a patient with failure of rendering the urine sugar-free [4].

Initially, the diminished insulin response was assumed to be related to fixed external factors that caused pancreatic insufficiency. These early reports of insulin resistance were among critically ill individuals with diabetes and comorbid conditions, including renal dysfunction, heart failure, infections, other endocrinopathies (pituitary and thyroid disease), tumors, and liver disease (cirrhosis and hemochromatosis), all now known to affect insulin sensitivity. In 1926, Mauriac and Aubertin also demonstrated that the insulin dose required to abolish glucosuria varied over time even in the same individual, suggesting that pancreatic function was "normal" in some cases and insulin was "neutralized" by other uncertain substances [4]. Simultaneously, others noted the association of glycosuria with stress hormones, such as epinephrine, corticotropin, and glucagon, supporting the hypothesis that other factors contributed to a reduced insulin response [7]. As refinements in insulin production yielded a purer product, these refractory insulin response reports became more numerous. However, data to support these theories were inconsistent. Multiple hypotheses were proposed. For example, a putative muscle enzyme, glycomutin, was believed to be a key mediator of insulin "neutralization" but was later discovered to be a contaminant [4]. To test the presence of substances that interfered with insulin action, patients' sera and insulin were injected into rabbits, and rabbit urine was monitored for glucosuria. However, these experiments resulted in a wide range of responses from severe convulsions (presumably secondary to hypoglycemia) and clearing of glucosuria to no effect. Therefore, the prevailing hypothesis soon after insulin's discovery was that there were 2 types of diabetes: one that resulted from pancreatic insufficiency and another that was related to a substance(s) that interfered with insulin action [4].

The first description of obesity-associated insulin resistance in 1927 was of a young man with excess fat stores, severe diabetes, and acidosis who had a reduction in glucosuria with a calorie-restricted diet but little to no response to injection of 160 units/day of insulin subcutaneously [5]. Notably, given that his obesity phenotype differed significantly from all prevailing reports of severely malnourished individuals with diabetes, he was not diagnosed with pancreatic diabetes; rather, his glucosuria was attributed to an alternative factor. The formal recognition of insulin resistance as a contributor to diabetes was not made until nearly a decade later. Himsworth systematically evaluated 2 groups of patients after a fixed oral glucose load before and after insulin administration. The insulin response was objectively measured, albeit indirectly, by comparing the change in urinary glucose concentrations between the 2 tests [8]. These experiments were significant because they were the first designed to quantify and standardize the insulin and glucose responses.

Following these clinical studies, Himsworth concluded that there were 2 distinct diabetes phenotypes. Individuals with "typical diabetes" (presumably due to insulin deficiency) were young and thin, had normal blood pressure, and severe sudden onset of hyperglycemia. The other group of individuals were older and had excess adiposity and comorbid conditions, such as hypertension and evidence of arteriosclerosis. The urinary glucose response differed markedly between the 2 groups; those who were older and had excess adiposity had higher urine glucose after a glucose load with or without fixed insulin administration (Fig. 1). Based on these studies, Himsworth proposed 2 types of diabetes: insulin-sensitive and insulininsensitive [9]. His proposal was accepted by the Royal College of Physicians of London in 1939, thereby acknowledging the existence of a biological phenomenon associated with a "diminished ability of the tissues to utilize glucose" $[10,11]$. The formal introduction of the term "insulin sensitivity" was monumental because the experiments provided novel and reproducible data that there were 2 pathophysiological causes of diabetes. 


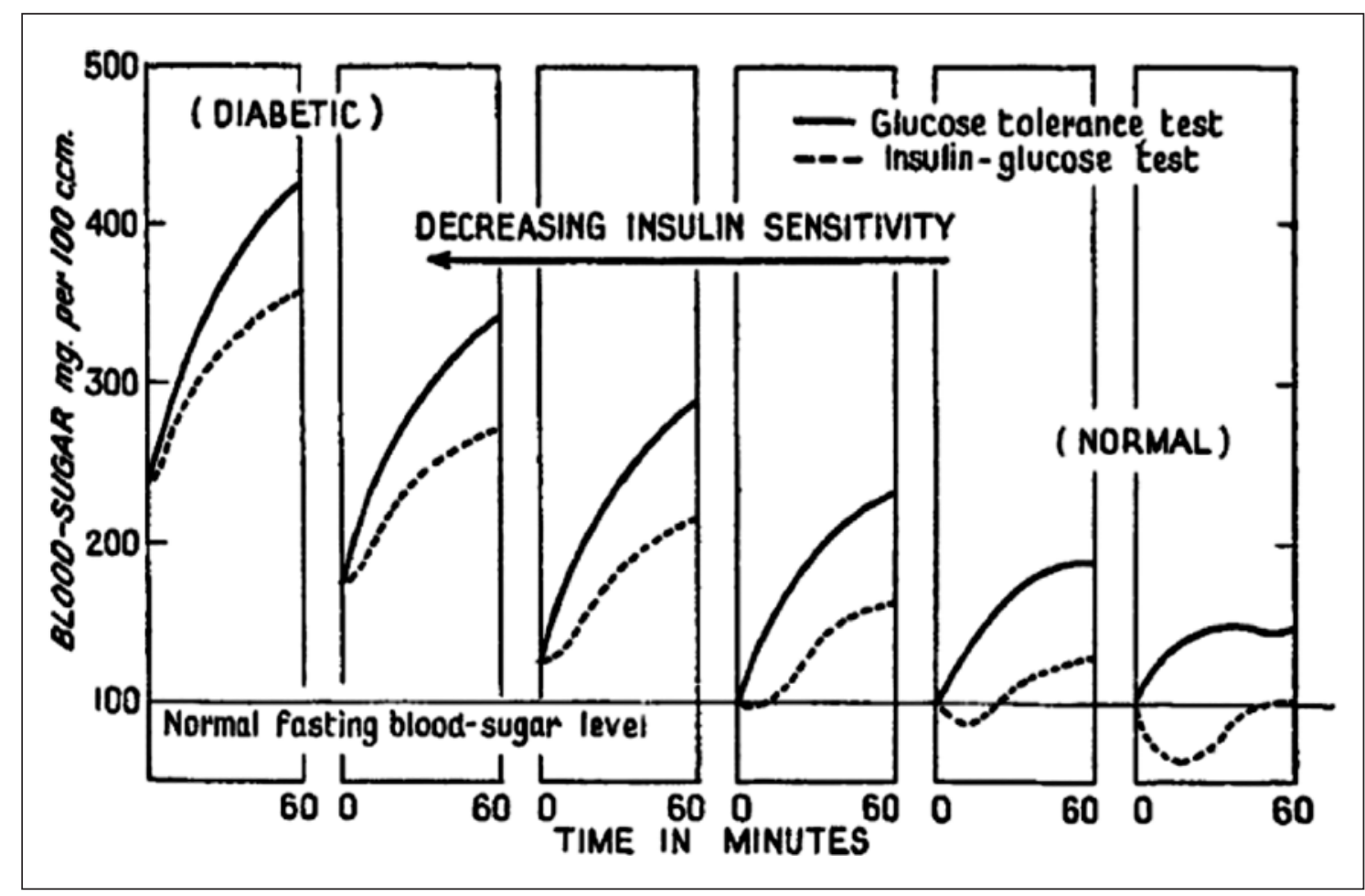

Fig. 1. Effect of decreasing insulin sensitivity on blood glucose tolerance and the insulin glucose curve in a group of individuals with normal glucose tolerance (right panels) to those with diabetes (left panels). The series of panels shows the progressive effect of decreasing insulin sensitivity on the blood glucose concentrations ( $y$ axis). Each panel depicts the average glucose curve for individuals who underwent two tests: a $60 \mathrm{~min}$ glucose tolerance test (solid lines) and a 60-min insulin glucose test (dashed lines). Adapted with permission from Himsworth [8].

\section{Evolution of Glucose and Insulin Assays Permitting Quantitation of Insulin Resistance}

Soon after the presentation to the Royal College of Physicians of London, attention quickly turned to developing insulin bioassays that would support the clinical phenotypes and observations that 2 different mechanisms may cause diabetes. Early methods, as previously descibed, were dependent on the ability of insulin to lower glucose in vivo or in vitro. In addition to evaluating the response to the oral glucose tolerance test, standard curves were also generated to quantify insulin sensitivity and indirectly estimate plasma insulin concentrations that would result in hypoglycemia in rats or rabbits [12, 13], glucose uptake in excised rat hemidiaphragms [14, 15], or oxidation rates of $\left[1-{ }^{14} \mathrm{C}\right]$ glucose in the rat epididymal fat pad [16]. Inherent to these initial experiments was the recognition that as individuals with diabetes ranged from insulin-sensitive to -insensitive, the same carbohydrate load resulted in higher blood glucose con- centrations. However, more precise quantification of this phenomenon termed "insulin insensitivity" was needed that did not rely on an indirect measurement of the glucose response to insulin administration or ex vivo standard curves. Critical next steps included the development of accurate glucose and insulin assays.

\section{Measurement of Glucose}

Qualitative methods for measuring glucose were first reported by Trommer (1841) and Fehling (1848) in urine and subsequently refined in the early 1900 s by utilizing various reducing substances that reacted with urine glucose [3]. However, these chemical reagents reacted with other compounds, including fructose, galactose, uric acid, ascorbic acid, ketone bodies, and salicylates. Although limited in specificity, these early methodologies paved the way for significant advances in point of care blood glucose measurements and stable laboratory-based analytical tools. Modern assays are enzymatic and specific and predominantly utilize glucose oxidase or hexo- 
kinase and a spectrophotometric indicator. Newer glucose assays have evolved over 4 generations of approaches: (1) reflectance meters; (2) modified sampling procedures with no wiping of the test strip, which reduced operator variation; (3) electrode technology/biosensor glucose meters; and most recently, (4) continuous glucose monitoring [3].

With the introduction of contemporary glucose assays, the factors that contributed to the documentation of insulin resistance became more readily apparent. From a research perspective, the introduction of the Yellow Springs Instrument glucose analyzer (YSI; Yellow Springs Instrument Co.) in 1975 marked a crucial milestone in glucose analytics, proving the existence of insulin resistance [3]. The YSI was the first successful, relatively portable tabletop machine that accurately and precisely measured blood glucose based on the oxidation and reduction of glucose and hydrogen peroxide using an oxygen electrode.

Researchers were then able to precisely quantify glucose response to a variety of insulin stimuli in a clinical metabolic research setting under near-steady-state conditions (e.g., during an insulin clamp) or during dynamic oral and intravenous glucose tolerance tests. Therefore, it is no surprise that the majority of experiments detailing the clinical and metabolic features of insulin resistance were published after the YSI became a "household" research tool [17].

The advent of continuous glucose monitoring systems is arguably one of the more revolutionary advancements in diabetes management as it facilitates timely and comprehensive blood glucose assessment without the need for multiple finger-sticks. These advanced technologies were important in the evolution of our understanding and treatment of insulin resistance as they resulted in improvements in self-glucose management and laboratory assessments of glucose concentrations.

\section{Measurement of Insulin}

History

Perhaps the most significant obstacle hindering the assessment of insulin resistance was the lack of a reliable insulin assay. Berson and Yalow's [18-20] sentinel breakthrough of identifying insulin-specific antibodies in insulin-treated patients was a direct consequence of the initial crude preparations of insulin, using nonhuman mammalian (porcine and bovine) pancreata, and the recognition that many, if not most, individuals with diabetes treated with these preparations developed antibodies to the foreign proteins. Using these antibodies, Berson and Yalow
[21-23] labeled the insulin protein with radioactive iodine and demonstrated that these antibodies could bind insulin, ultimately leading to the development of the RIA to measure small amounts of insulin in biological samples. In brief, animals (usually guinea pigs) were immunized with insulin plus Freund's adjuvant (the latter boosted the immune response), and plasma insulin was estimated using a competitive binding assay of unlabeled and radiolabeled insulin. The antibody-bound insulin was precipitated with a second antibody directed against the first antibody complex (e.g., rabbit anti-guinea pig gamma globulin). The more unlabeled insulin in the reaction tube, the less labeled insulin was bound to the first antibody and thus in the second antibody precipitated complex. Patient sample assays were run in parallel with a standard curve of purified insulin to calculate the concentration of insulin in the unknown sample (Fig. 2) [22$24]$. Distinct phenotypes of diabetes could now be ascertained based on combining clinical characteristics with measurements of serum glucose and insulin concentrations during standardized oral glucose tolerance tests [25]. Subsequently, advances in the RIA occurred, in part, because of innovative methods that were developed to separate the antibody-bound and free hormones [26]. Later, this methodology led to a new revolution in measuring a variety of circulating hormones (e.g., parathyroid- and thyroid-stimulating hormones).

\section{Measurement Challenges and Insulin Antibodies}

Notably, several analytical and biological factors should be considered, especially when assessing fasting insulin concentrations that are physiologically present in very low concentrations. Analytical issues include the type and processing of biological samples and the analytical platform utilized. Standardizing sample collection is critical because hemolyzed samples may result in systematic underestimation of plasma insulin concentrations. When comparing experiments, it is also important to note the sample collection type as plasma insulin concentrations are lower than serum measurements. Today, multiple insulin assays are available, but there is no gold standard technique for measuring insulin; the conspicuous lack of standardization across assays makes a direct comparison between studies and populations difficult [26].

The most important biological factor that should be considered in assessing insulin resistance and its measurement is the occurrence of antibodies directed to endogenous or exogenous insulin [27]. Radio- and enzymelinked immunoassays work well for quantifying insulin in 
Fig. 2. a Radiochromato-electrophoretograms of antiserum and insulin mixtures. Mixtures contained the same concentrations of guinea pig antibeef insulin serum and beef insulin-I131 but varying concentrations of human insulin as indicated. Adapted with permission from Yalow et al. [22]. b Ratio of bound insulin-I131 to free insulin-I131 as a function of concentration of added human insulin. The ratios were obtained from the complete series of radiochromato-electrophoretograms as shown in a. Adapted with permission from Yalow et al. [22].
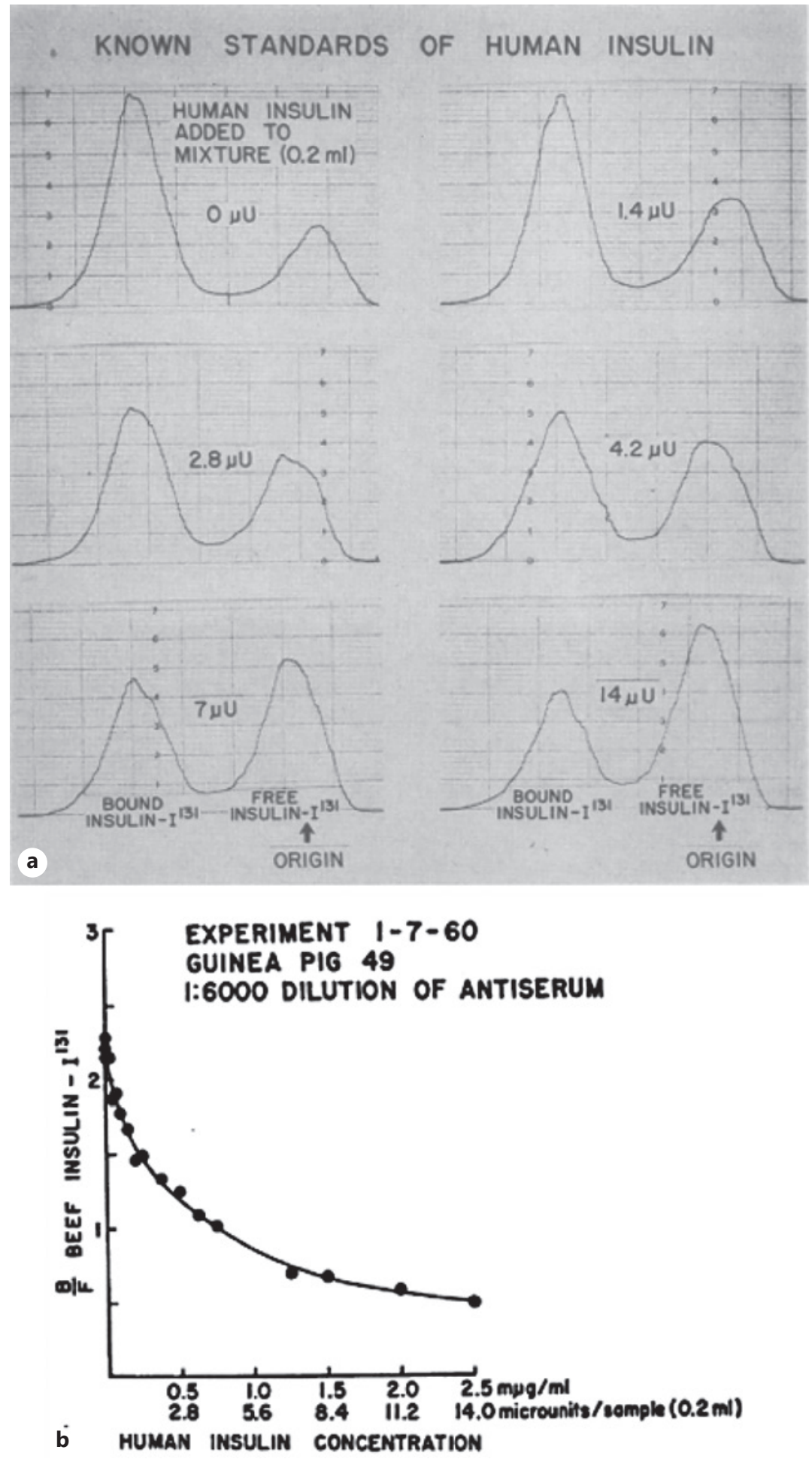
individuals never exposed to exogenous insulin but may be less accurate in insulin-treated patients because of the potential of endogenous insulin antibodies and degraded insulin products that may interfere with antibody-antigen interaction [26]. Simple mathematical corrections to account for endogenous insulin antibodies are not possible because of inherent differences in insulin antibody concentrations and affinity. In addition, the discovery of anti-insulin antibodies in insulin-naive individuals with new-onset type 1 diabetes gave insights into its pathophysiology but also interfered with the quantitation of insulin in these individuals [28]. Newer analytical techniques, such as immuno-capture tandem mass spectrometry, have a wide quantitative range, precision, and accuracy. However, inaccuracies caused by circulating antibodies will most likely remain [29]. Readers should carefully scrutinize methodologies and results of "free" insulin concentrations that attempt to correct for the presence of insulin-bound to anti-insulin antibodies.

The evolution of purified mammalian insulin reduced the frequency of insulin antibodies. Initially, this relied on further purification of the pancreatic extracts using liquid chromatography (e.g., monocomponent or single-peak insulin) and then humanized insulin. The first insulin preparations used in humans required frequent daily injections (4-8 times daily) but were soon followed by nextgeneration products such as protamine zinc insulin (Ultra-lente in 1935), a long-acting insulin, and Neutral Protamine Hagadorn (1950), an intermediate acting insulin. Several crucial steps had to be developed before additional advances in the drug, insulin, could be made. These included the determination of the amino acid sequence of purified human insulin, the description of the gene sequence of the proinsulin gene, the insertion of the gene into an E. Coli vector, the development of fermentation processes to grow the $E$. coli containing the insulin gene that resulted in the production of proinsulin, the pasteurization of the product, the cleavage of the connecting peptide (C-peptide), and then finally, isolation and purification of human insulin produced in E. coli $[1,30]$. This was followed by carefully conducted clinical trials and finally the FDA approval of the first recombinant human drug in 1982, marking the birth of biotechnology [31]. The advent of recombinant insulin significantly reduced but did not eliminate the presence of insulin antibodies [1]. Subsequently, genetic engineering to slightly but significantly alter the insulin amino acid sequences resulted in profound effects on the pharmacologic action of the native recombinant human insulin. Therefore, most modern-day assays can measure total endogenous insulin without interference from insulin analogs, and vice versa. These modifications have again made the measurement of insulin more complex to the point that specific assays will only measure a specific new recombinant hormone reliably and only weakly cross-react with endogenous insulin. Only a polyclonal antibody assay will measure the total "insulin" in a sample containing both native human and recombinant insulin. This is of particular importance in patients with Munchausen or Munchausen by proxy in which the spuriously injected insulin will most like not be native recombinant human insulin but a more common insulin analog.

\section{Contemporary Quantitation of Insulin Resistance in Humans with Obesity and Diabetes}

After establishing a relatively reliable method for assessing insulin concentrations, the focus shifted to defining the insulin resistance syndrome(s) and its association with obesity, diabetes, and other chronic diseases. Perley and Kipnis were among the first to undertake classic studies measuring insulin secretion and resistance in individuals with diabetes and obesity [32]. Plasma glucose concentrations were carefully matched in 4 different groups (normal-weight and obese without diabetes and normalweight and obese with diabetes) during oral or intravenous glucose administration. These elegant studies demonstrated 2 key pathological features of obesity and diabetes (Fig. 3) [32]. First, insulin response was lower in individuals with diabetes than those without, regardless of the route of glucose administration or whether the individual was lean or obese. Second, individuals with obesity had higher insulin responses, despite similar glucose concentrations, than their normal weight peers, which led the authors to conclude that "the presence of significant insulin antagonism [was] associated with obesity per se" [32]. Over time, incremental improvements in estimating insulin resistance led to innovations, providing various new models and methodologies.

\section{Modern Measurements of Insulin Resistance (Table 1)}

Over the last 3 decades, numerous additional approaches emerged for quantifying insulin, ranging from fasting single blood sample indices to provocative and dynamic tests. Table 1 gives a high-level summary of the common tests available, their advantages and limitations, and, when available, correlations with the gold standard 


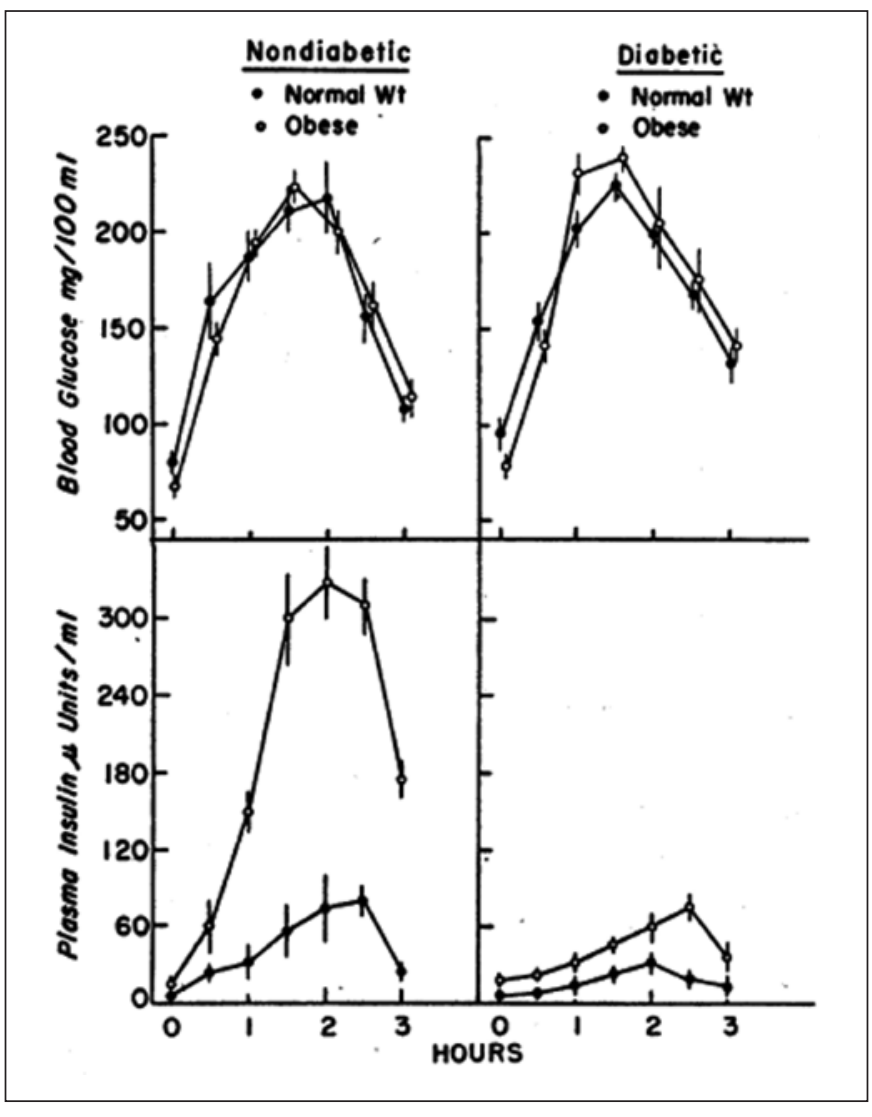

Fig. 3. Plasma glucose and insulin responses of individuals with and without diabetes and obesity to glucose infusion to maintained diabetic blood glucose profiles. Left panel: 12 normal-weight (filled circles) and 12 obese without diabetes (open circles). Right panel: 12 normal-weight with diabetes (filled circles) and 11 obese without diabetes (open circles). Data represent mean \pm standard errors. Adapted with permission from Perley et al. [32].

euglycemic hyperinsulinemic clamp. (For a detailed discussion of each test, we refer to the reader to current, indepth reviews [33-36].) Each of these techniques has its own set of assumptions and limitations. Therefore, choosing the most appropriate test for assessing insulin resistance depends on the study design and population, available clinical and research resources, and the underlying hypotheses or expected clinical outcome.

The development of the euglycemic hyperinsulinemic and the hyperglycemic clamps provided new and exciting tools for assessing insulin resistance in vivo. The euglycemic hyperinsulinemic clamp facilitated precise quantification of glucose disposal relative to a fixed plasma concentration of insulin under steady-state conditions that is utilized across a wide range of human and animal models. In addition, when used with simultaneous infusion of so- matostatin, recombinant growth hormone, and glucagon, it has the potential advantage of controlling for counter-regulatory hormones that are known to interfere with tissue glucose responsiveness [37]. The euglycemic hyperinsulinemic clamp is considered the gold standard for measuring insulin resistance, and its use has corroborated the role of insulin resistance in a variety of diseases including obesity, type 2 diabetes mellitus, atherosclerotic disease, polycystic ovarian syndrome, nonalcoholic fatty liver disease, $\mathrm{CKD}$, inflammatory disorders, and drugs (e.g., corticosteroids) [17]. The hyperglycemic clamp technique, raising the plasma glucose concentration to near or at the renal threshold, has the additional advantage of providing an estimate of maximal insulin secretion and maximal rates of glucose utilization.

Although the hyperinsulinemic euglycemic insulin clamp provides the most reproducible results, it is a nonphysiologic test that is operator-dependent, time-consuming, expensive, and limited to populations that can tolerate the procedure safely even with appropriate assent or consent (Table 1). Because of these factors, its use is limited to relatively small numbers of subjects and it is rarely used in young children and infants. As a result, numerous less invasive approximations have evolved including intravenous and oral tolerance tests. Yet, none of these techniques have been adopted as a clinical tool for measuring insulin resistance because the tests still require specialized expertise, resources, tools, models, and accurate measures of insulin across laboratories and analytical platforms.

Single-sample biochemical markers, especially the homeostasis model of insulin resistance (HOMA-IR and HOMA2), are clinically attractive because they are easy to obtain. However, there is marked interindividual variability with only moderate agreement with the hyperinsulinemic euglycemic clamp which may be related to wide interindividual variability because of the lack of standardization of insulin assays across platforms and/or altered relationships of insulin concentration with insulin resistance in individuals with diabetes. Therefore, the HOMA models are most suitable for population assessments as an epidemiological tool. Novel markers that are promising additions to the clinician's toolbox are surrogate indices of insulin resistance obtained after single blood drawn. Specifically, a nuclear magnetic resonance biomarker, lipoprotein insulin resistance (LPIR) index, derived from deconvolution of methyl lipid spectra, reflects a nonglycemic measure of insulin resistance. The LPIR index has the added advantage of improving risk prediction for type 2 diabetes and related complications, which 


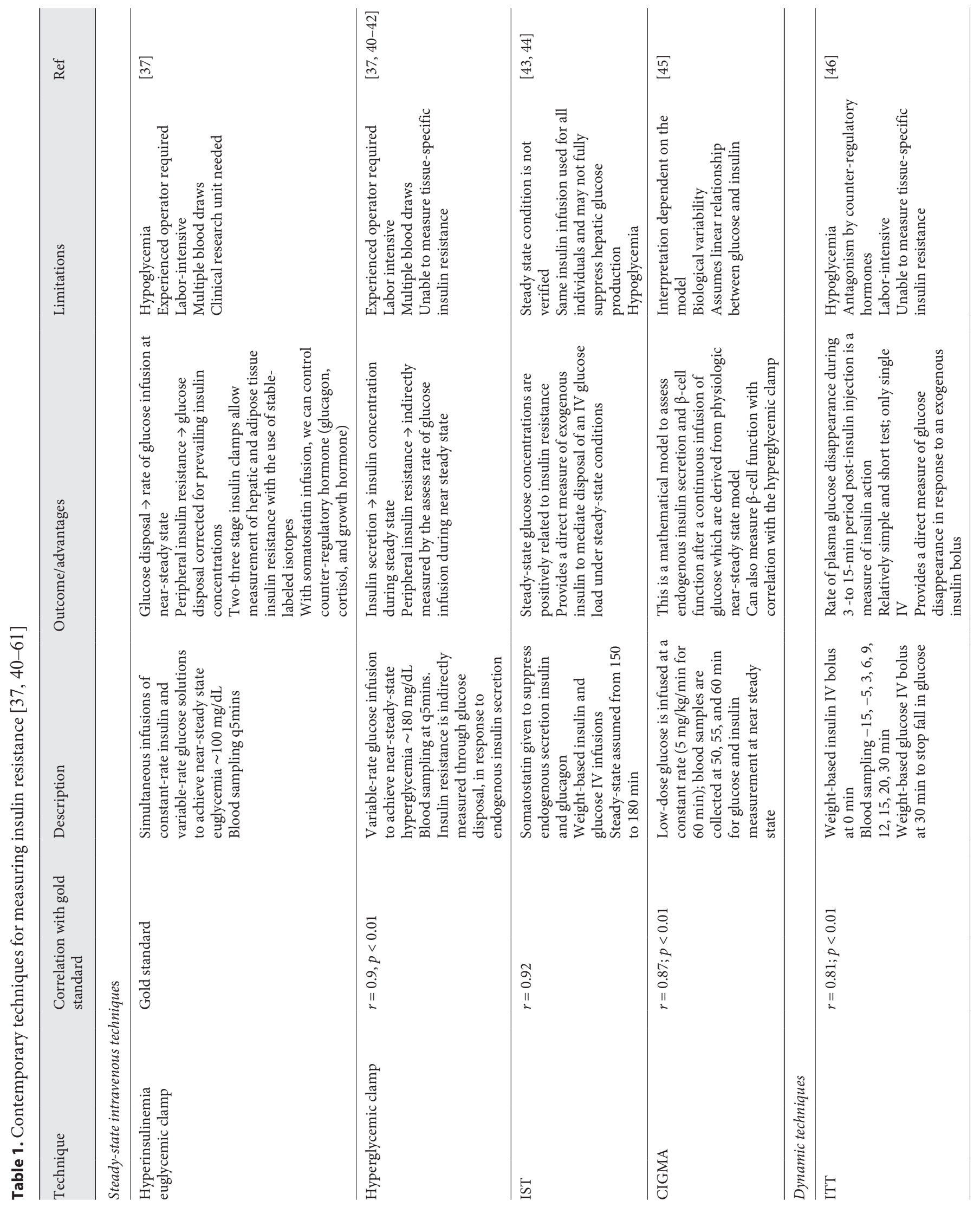




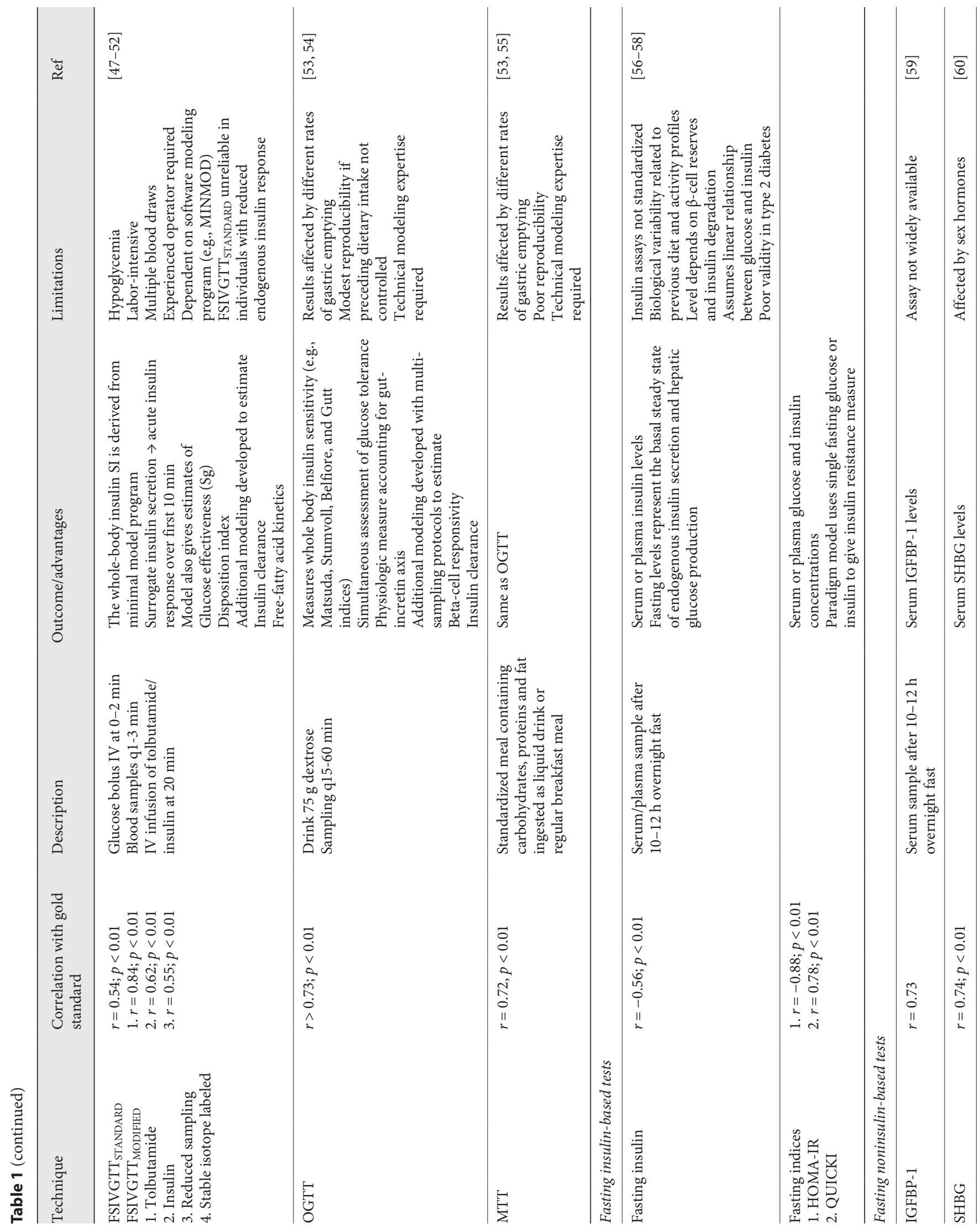




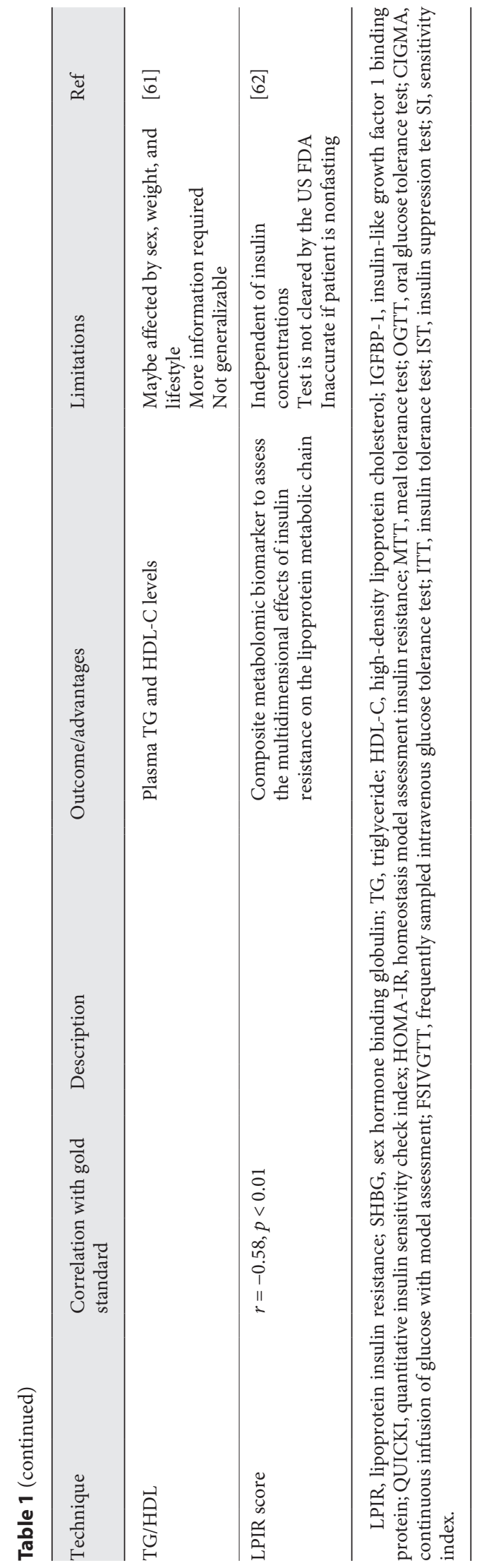

would be valuable information for the busy clinician [38, 39]. Such techniques are not widely available nor accepted but offer promise for future assessments that will combine the convenience and accuracy needed for clinical care balanced with the cost and resources needed for widespread distribution and standardization.

\section{Conclusions}

Insulin resistance is a pathophysiological entity that has evolved to be a global public health problem associated with a myriad of chronic metabolic diseases. Addressing this worldwide challenge requires accurate quantification, risk prediction, and assessment of insulin resistance, including critical evaluation of how insulin resistance measures change with interventions. In this historical perspective, we demonstrated how each of the advances in insulin and glucose analytics contributed to the recognition and validation of insulin resistance. As we move forward to the next 100 years of insulin, we must focus on primary and secondary interventions for insulin resistance and anticipate improved translational techniques that will support these efforts across the metabolic disease spectrum.

\section{Acknowledgements}

We would like to thank Celeste Cravalho for her editorial assistance.

\section{Conflict of Interest Statement}

I, S.T.C., certify that neither I nor my coauthors have any conflicts of interest.

\section{Funding Sources}

The Division of Intramural Research, National Institute of Diabetes and Digestive and Kidney Diseases, National Institutes of Health supported S.T.C.

\section{Author Contributions}

S.T.C., V.R.S., and M.W.H. conceptualized, performed the literature search, and wrote and edited the manuscript. S.T.M. performed the literature search and edited the manuscript. All authors reviewed and approved the final version of the manuscript. 


\section{References}

1 Hirsch IB, Juneja R, Beals JM, Antalis CJ, Wright EE. The evolution of insulin and how it informs therapy and treatment choices. Endocr Rev. 2020;41(5):bnaa015.

2 Vecchio I, Tornali C, Bragazzi NL, Martini M. The discovery of insulin: an important milestone in the history of medicine. Front Endocrinol. 2018;9:613.

3 Moodley N, Ngxamngxa U, Turzyniecka MJ, Pillay TS. Historical perspectives in clinical pathology: a history of glucose measurement. J Clin Pathol. 2015;68(4):258-64.

4 Root HF. Insulin resistance and bronze diabetes. N Engl J Med. 1929;201(5):201-6.

5 Glassberg BY, Somogyi M, Taussig AE. Diabetes mellitus. Arch Intern Med. 1927;40(5): 676-85.

6 Macleod JJR. Insulin and diabetes. A general statement of the physiological and therapeutic effects of insulin. British Medical Journal. 1922;1922:833-5.

7 Falta W, Boller R. Insulärer und Insulinresistenter Diabetes. Klin Wochenschr. 1931; 10(10):438-43

8 Himsworth HP. The mechanism of diabetes mellitus: III - Human diabetes mellitus. Lancet. 1939;234(6047):171-6.

9 Himsworth HP. Diabetes mellitus. Lancet. 1936;227(5864):127-30.

10 Himsworth HP. Insulin resistance. Lancet. 1939;234(6050):395.

11 Himsworth P, Kerr RB. Insulin sensitive and insulin-insensitive types of diabetes mellitus. Clin Sci. 1939;4(2):119-52.

12 Bornstein J, Lawrence RD. Plasma insulin in human diabetes mellitus. Br Med J. 1951; 2(4747):1541-4.

13 Berson SA, Weisenfeld S, Pascullo M. Utilization of glucose in normal and diabetic rabbits; effects of insulin, glucagon and glucose. Diabetes. 1959;8(2):116-27.

14 Groen J, Kamminga CE, Willebrands AF, Blickman JR. Evidence for the presence of insulin in blood serum; a method for an approximate determination of the insulin content of blood. J Clin Invest. 1952;31(1):97-106.

15 Baird CW, Bornstein J. Plasma-insulin and insulin resistance. Lancet. 1957;272(6979): 1111-3.

16 Martin DB, Renold AE, Dagenais YM. An assay for insulin-like activity using rat adipose tissue. Lancet. 1958;2(7037):76-7.

17 Petersen MC, Shulman GI. Mechanisms of in sulin action and insulin resistance. Physio Rev. 2018;98(4):2133-223.

18 Berson SA, Yalow RS. Species-specificity of human antibeef, pork insulin serum. J Clin Invest. 1959;38:2017-25.

19 Berson SA, Yalow RS. Quantitative aspects of the reaction between insulin and insulinbinding antibody. J Clin Invest. 1959;38: 1996-2016.

20 Berson SA, Yalow RS. Recent studies on insulin-binding antibodies. Ann N Y Acad Sci. 1959;82:338-44.
21 Yalow RS, Berson SA. Assay of plasma insulin in human subjects by immunological methods. Nature. 1959;184(Suppl 21):1648-9.

22 Yalow RS, Berson SA. Immunoassay of endogenous plasma insulin in man. J Clin Invest. 1960;39(7):1157-75.

23 Yalow RS, Berson SA. Plasma insulin concentrations in nondiabetic and early diabetic subjects. Determinations by a new sensitive immuno-assay technic. Diabetes. 1960;9:25460.

24 Yalow RS, Black H, Villazon M, Berson SA. Comparison of plasma insulin levels following administration of tolbutamide and glucose. Diabetes. 1960;9:356-62.

25 Reaven G, Miller R. Study of the relationship between glucose and insulin responses to an oral glucose load in man. Diabetes. 1968; 17(9):560-9.

26 Shen Y, Prinyawiwatkul W, Xu Z. Insulin: a review of analytical methods. Analyst. 2019; 144(14):4139-48.

27 Samaan N, Fraser R, Dempster WJ. The "typical" and atypical" forms of serum insulin. Diabetes. 1963;12:339-48.

28 Singal DP, Blajchman MA. Histocompatibility (HL-A) antigens, lymphocytotoxic antibodies and tissue antibodies in patients with diabetes mellitus. Diabetes. 1973;22(6):42932.

29 Taylor SW, Clarke NJ, Chen Z, McPhaul MJ. A high-throughput mass spectrometry assay to simultaneously measure intact insulin and C-peptide. Clin Chim Acta. 2016;455:202-8.

30 Goeddel DV, Kleid DG, Bolivar F, Heyneker HL, Yansura DG, Crea R, et al. Expression in Escherichia coli of chemically synthesized genes for human insulin. Proc Natl Acad Sci USA. 1979;76(1):106-10.

31 Junod S.W., Celebrating a milestone: FDA's approval of first genetically-engineered product. 2007. U.S.F.D. Administration, Editor. US FDA: History Corner Update Magazine.

32 Perley MJ, Kipnis DM. Plasma insulin responses to oral and intravenous glucose: studies in normal and diabetic sujbjects. J Clin Invest. 1967;46(12):1954-62.

33 Brown RJ, Yanovski JA. Estimation of insulin sensitivity in children: methods, measures and controversies. Pediatr Diabetes. 2014; 15(3):151-61.

34 Antuna-Puente B, Disse E, Rabasa-Lhoret R, Laville M, Capeau J, Bastard JP. How can we measure insulin sensitivity/resistance?. Diabetes Metab. 2011;37(3):179-88.

35 Borai A, Livingstone C, Ferns GA. The biochemical assessment of insulin resistance. Ann Clin Biochem. 2007;44(Pt 4):324-42.

36 Muniyappa R, Lee S, Chen H, Quon MJ. Current approaches for assessing insulin sensitivity and resistance in vivo: advantages, limitations, and appropriate usage. Am J Physiol Endocrinol Metab. 2008;294(1):E15-26.
37 DeFronzo RA, Tobin JD, Andres R. Glucose clamp technique: a method for quantifying insulin secretion and resistance. Am J Physiol. 1979;237(3):E214-23.

38 Flores-Guerrero JL, Connelly MA, Shalaurova I, Gruppen EG, Kieneker LM, Dullaart $\mathrm{RPF}$, et al. Lipoprotein insulin resistance index, a high-throughput measure of insulin resistance, is associated with incident type II diabetes mellitus in the Prevention of Renal and Vascular End-Stage Disease study. J Clin Lipidol. 2019 Jan-Feb;13(1):129-e1.

39 Harada PHN, Demler OV, Dugani SB, Akinkuolie AO, Moorthy MV, Ridker PM, et al. Lipoprotein insulin resistance score and risk of incident diabetes during extended follow-up of 20 years: the Women's Health Study. J Clin Lipidol. 2017;11(5) 1257-e2.

40 Sjaarda L, Lee S, Tfayli H, Bacha F, Bertolet M, Arslanian $\mathrm{S}$ et al. Measuring beta-cell function relative to insulin sensitivity in youth does the hyperglycemic clamp suffice? Diabetes Care. 2013;36(6):1607-12.

41 Hannon TS, Kahn SE, Utzschneider KM, Buchanan TA, Nadeau KJ, Zeitler PS et al. Review of methods for measuring beta-cell function: design considerations from the Restoring Insulin Secretion (RISE) Consortium. Diabetes Obes Metab. 2018;20(1):1424.

42 Elahi D. In praise of the hyperglycemic clamp. A method for assessment of beta-cell sensitivity and insulin resistance. Diabetes Care. 1996;19(3):278-86.

43 Greenfield MS, Doberne L, Kraemer F, Tobey $\mathrm{T}$, Reaven G. Assessment of insulin resistance with the insulin suppression test and the euglycemic clamp. Diabetes. 1981;30(5):38792.

44 Shen SW, Reaven GM, Farquhar JW. Comparison of impedance to insulin-mediated glucose uptake in normal subjects and in subjects with latent diabetes. J Clin Invest. 1970; 49(12):2151-60.

45 Hosker JP, Matthews DR, Rudenski AS, Burnett MA, Darling P, Bown EG, et al. Continuous infusion of glucose with model assessment: measurement of insulin resistance and beta-cell function in man. Diabetologia. 1985; 28(7):401-11.

46 Bonora E, Moghetti P, Zancanaro C, Cigolini M, Querena M, Cacciatori V, et al. Estimates of in vivo insulin action in man: comparison of insulin tolerance tests with euglycemic and hyperglycemic glucose clamp studies. J Clin Endocrinol Metab. 1989;68(2):374-8.

47 Pacini G, Bergman RN. MINMOD: a computer program to calculate insulin sensitivity and pancreatic responsivity from the frequently sampled intravenous glucose tolerance test. Comput Methods Programs Biomed. 1986;23(2):113-22. 
48 Polidori DC, Bergman RN, Chung ST, Sumner AE. Hepatic and extrahepatic insulin clearance are differentially regulated: results from a novel model-based analysis of intravenous glucose tolerance data. Diabetes. 2016; 65(6): 1556-64.

49 Saad MF, Anderson RL, Laws A, Watanabe RM, Kades WW, Chen YD, et al. A comparison between the minimal model and the glucose clamp in the assessment of insulin sensitivity across the spectrum of glucose tolerance. Insulin Resistance Atherosclerosis Study. Diabetes. 1994;43(9):1114-21.

50 Sumner AE, Luercio MF, Frempong BA, Ricks M, Sen S, Kushner H, et al. Validity of the reduced-sample insulin modified frequently-sampled intravenous glucose tolerance test using the nonlinear regression approach. Metab Clin Exp. 2009;58(2):220-5.

51 Caumo A, Giacca A, Morgese M, Pozza G, Micossi P, Cobelli C. Minimal models of glucose disappearance: lessons from the labelled IVGTT. Diabet Med. 1991;8(9):822-32.

52 Beard JC, Bergman RN, Ward WK, Porte D. The insulin sensitivity index in non-diabetic man: correlation between clampderived and IVGTT-derived values. Diabetes 1986;35:3629.
53 Cobelli C, Toffolo GM, Dalla Man C, Campioni M, Denti P, Caumo A, et al. Assessment of beta-cell function in humans, simultaneously with insulin sensitivity and hepatic extraction, from intravenous and oral glucose tests. Am J Physiol Endocrinol Metab. 2007; 293(1):E1-E15.

54 Matsuda M, DeFronzo RA. Insulin sensitivity indices obtained from oral glucose tolerance testing: comparison with the euglycemic insulin clamp. Diabetes Care. 1999;22(9):1462-70.

55 Dalla Man C, Piccinini F, Basu R, Basu A, Rizza RA, Cobelli C. Modeling hepatic insulin sensitivity during a meal: validation against the euglycemic hyperinsulinemic clamp. Am J Physiol Endocrinol Metab. 2013;304(8): E819-25.

56 Matthews DR, Hosker JP, Rudenski AS, Naylor BA, Treacher DF, Turner RC. Homeostasis model assessment: insulin resistance and beta-cell function from fasting plasma glucose and insulin concentrations in man. Diabetologia. 1985;28(7):412-9.

57 Wallace TM, Levy JC, Matthews DR. Use and abuse of HOMA modeling. Diabetes Care. 2004;27(6):1487-95.
58 Katz A, Nambi SS, Mather K, Baron AD, Follmann DA, Sullivan G, et al. Quantitative insulin sensitivity check index: a simple, accurate method for assessing insulin sensitivity in humans. J Clin Endocrinol Metab. 2000; 85(7):2402-10.

59 Maddux BA, Chan A, De Filippis EA, Mandarino LJ, Goldfine ID. IGF-binding protein-1 levels are related to insulin-mediated glucose disposal and are a potential serum marker of insulin resistance. Diabetes Care. 2006;29(7): $1535-7$.

60 Birkeland KI, Hanssen KF, Torjesen PA, Vaaler S. Level of sex hormone-binding globulin is positively correlated with insulin sensitivity in men with type 2 diabetes. J Clin Endocrinol Metab. 1993;76(2):275-8.

61 Karelis AD, Pasternyk SM, Messier L, StPierre DH, Lavoie JM, Garrel D, et al. Relationship between insulin sensitivity and the triglyceride-HDL-C ratio in overweight and obese postmenopausal women: a MONET study. Appl Physiol Nutr Metab. 2007;32(6): 1089-96.

62 Shalaurova I, Connelly MA, Garvey WT, Otvos JD. Lipoprotein insulin resistance index: a lipoprotein particle-derived measure of insulin resistance. Metab Syndr Relat Disord. 2014;12(8):422-9. 